\title{
Effect of diameter of nanoparticles and capture cross-section library on macroscopic dose enhancement in boron neutron capture therapy
}

\author{
Bagher Farhood, MSc, Mahdi Ghorbani, PhD \\ Medical Physics Department, Faculty of Medicine, Mashhad University of Medical Sciences, Mashhad, Iran
}

\begin{abstract}
Purpose: The aim of this study is evaluation of the effect of diameter of ${ }^{10} \mathrm{~B}$ nanoparticles and various neutron capture cross-section libraries on macroscopic dose enhancement in boron neutron capture therapy (BNCT).

Material and methods: MCNPX Monte Carlo code was used for simulation of a ${ }^{252} \mathrm{Cf}$ source, a soft tissue phantom and a tumor containing ${ }^{10} \mathrm{~B}$ nanoparticles. Using ${ }^{252} \mathrm{Cf}$ as a neutron source, macroscopic dose enhancement factor (MDEF) and total dose rate in tumor in the presence of 100, 200, and $500 \mathrm{ppm}$ of ${ }^{10} \mathrm{~B}$ nanoparticles with $25 \mathrm{~nm}, 50 \mathrm{~nm}$, and $100 \mathrm{~nm}$ diameters were calculated. Additionally, the effect of ENDF, JEFF, JENDL, and CENDL neutron capture cross-section libraries on MDEF was evaluated.

Results: There is not a linear relationship between the average MDEF value and nanoparticles' diameter but the average MDEF grows with increased concentration of ${ }^{10} \mathrm{~B}$ nanoparticles. There is an increasing trend for average MDEF with the tumor distance. The average MDEF values were obtained the same for various neutron capture cross-section libraries. The maximum and minimum doses that effect on the total dose in tumor were neutron and secondary photon doses, respectively. Furthermore, the boron capture related dose component reduced in some extent with increase of diameter of ${ }^{10} \mathrm{~B}$ nanoparticles.

Conclusions: Based on the results of this study, it can be concluded that from physical point of view, various nanoparticle diameters have no dominant effect on average MDEF value in tumor. Furthermore, it is concluded that various neutron capture cross-section libraries are resulted to the same macroscopic dose enhancements. However, it is predicted that taking into account the biological effects for various nanoparticle diameters will result in different dose enhancements.
\end{abstract}

Key words: boron neutron capture therapy, cross-section library, dose enhancement nanoparticle.

\section{Purpose}

Boron neutron capture therapy (BNCT) was first described in 1935 [1]. Boron neutron capture therapy is a biochemically targeted radiation therapy based on the nuclear capture that happens when non-radioactive ${ }^{10} \mathrm{~B}$ is irradiated with thermal neutrons $(0.025 \mathrm{eV})$. This interaction results in high linear energy transfer products (alpha particles and recoiling lithium-7 nuclei) [2]. These heavy particles deposit their energy $(2.34 \mathrm{MeV})$ in range of 5-9 $\mu \mathrm{m}$, which corresponds to cell diameter [3]. The destructive effects of these particles are restricted to boron containing cells. Since these heavy particles have very short path lengths in tissue, BNCT provides a way to selectively destroy cancer cells and spare adjacent normal cells [4]. Clinical applications of BNCT consist of treatment of gliomas [5], melanoma [6], liver cancer [7], colon cancer [8], sarcoma [9], lung cancer, mesothelioma, chest tumors [10], and head and neck tumors [11].
Despite recent improvements in conventional cancer treatment modalities, there is a significant need for new treatments that can destroy malignant cells while causing much less damage to the normal cells $[12,13]$. For this purpose, researchers are making effort to incorporate nanotechnology into present cancer therapeutics and imaging. The final goal of use of nanoparticles will be targeted delivery to the tumor, while causing to the least side effects for other tissues [14-16].

There are numerous studies, which have been carried out in the field of use of nanoparticles as dose enhancment media in radiotherapy. Some of these studies are reviewed herein: Ghorbani et al. [17] in a Monte Carlo study, have evaluated the effect of diameter of gold nanoparticles and method of modeling on macroscopic dose enhancement in brachytherapy. The results of their study indicated that with gold nanoparticles of larger diameter, the amount of macroscopic dose enhancement inside the tumor is higher. 
It has been also demonstrated that a simple model overestimates the dose macroscopic enhancement when compared with an exact model, in which nanoparticles are defined precisely in the Monte Carlo code. Pakravan et al. [18] in a Monte Carlo study have investigated the size effect of gold nanoparticles on macroscopic tumor dose enhancement in a $6 \mathrm{MeV}$ photon beam. In the study, they found that there is a negligible relation between the dose enhancement and nanoparticles' diameter. It was also evident that for high energy photons, the macroscopic dose enhancement is less affected by the diameter of nanoparticles than their concentration.

Ghassoun et al. [19], in a Monte Carlo study, have evaluated dose distribution of a ${ }^{252} \mathrm{Cf}$ brachytherapy source in BNCT. The results indicated that the magnitude of dose increase would depend on the distance from the ${ }^{252} \mathrm{Cf}$ source as well as the concentration of ${ }^{10} \mathrm{~B}$ in the tumor cells. They found that a significant increase in dose is gained for tumor cells located at several centimeters away from the source with higher ${ }^{10} \mathrm{~B}$ loadings. Rivard and Zamenhof [20] carried out Monte Carlo calculations on a brain phantom to assess the boron-neutron capture dose and the ${ }^{252} \mathrm{Cf}$ neutron energy spectra. ${ }^{10} \mathrm{~B}$ loadings up to 50 ppm did not noticeably modify the ${ }^{252} \mathrm{Cf}$ fast neutron energy spectrum. For ${ }^{10} \mathrm{~B}$ concentrations lower than $50 \mathrm{ppm}$, the neutron dose emitted from ${ }^{252} \mathrm{Cf}$ was small compared to the fast neutron sources. Finally, ${ }^{252} \mathrm{Cf}$ could be clinically useful as a neutron source for boron neutron capture enhanced brachytherapy but would require the use of improved ${ }^{10} \mathrm{~B}$ agents with better loadings and excellent tumor selectivity.

Sumitani and Nagasaki [21] have studied BNCT assisted by boron-conjugated nanoparticles. In their study, they indicated that core-polymerized and boron-conjugated micelles administered via intravenous injection in tumor were averted almost entirely from the major organs, except for the tumor, after a week. Accordingly, boron-conjugated micelles indicated a promising approach for development of a new boron carrier for BNCT. Hwang et al. [22] have studied neutron capture nuclei containing carbon nanoparticles. In the study, they have demonstrated examples for neutron capture therapy (NCT) such as: BNCT, GdNCT, and CoNCT at the cellular level, in which ${ }^{10} \mathrm{~B},{ }^{157} \mathrm{Gd}$, and ${ }^{59} \mathrm{Co}$ containing carbon nanoparticles could significantly induce acute cell deaths following receiving thermal neutron irradiation. The medical efficacies of the carbon nanoparticles can be better optimized by use of pure ${ }^{157} \mathrm{Gd}$ or ${ }^{10} \mathrm{~B}$ isotopes. The results indicated that these neutron capture elements containing carbon nanoparticles are very promising medical nanomaterials for destruction of tumors. In another NCT Monte Carlo study by Porras [23], the potential effect of enhancement of BNCT in presence of ${ }^{33} \mathrm{~S}$ nanoparticles was investigated. The results showed that a noticeable local enhancement in dose could be obtained by means of sulfur nanoparticles. This effect may be synergic to the boron addition for NCT. This method can be considered as an alternative to the tailoring of neutron spectrum with the advantage of not increasing the gamma component of the background tissue dose.
Recently, in a report by International Atomic Energy Agency (IAEA) on various cross-section standards for neutron interactions, it was discussed that there are differences in neutron cross-section values at different neutron energies due to the related uncertainties in the measurements [24]. Since neutron capture cross-section libraries are used for calculation of neutron capture macroscopic dose enhancement, the use of different cross-section libraries should be investigated to answer this question that "has cross-section library an effect on macroscopic dose enhancement factor value?".

To the best of our knowledge, there is not any investigation on the effect of diameter of nanoparticles and neutron capture cross-section library on macroscopic dose enhancement in BNCT. In the present study, macroscopic dose enhancement for various diameters of ${ }^{10} \mathrm{~B}$ nanoparticles and various neutron capture cross-section libraries was evaluated and compared with each other.

\section{Material and methods}

Monte Carlo simulations were performed, while a ${ }^{252} \mathrm{Cf}$ source $[22,25]$ was defined as the source of neutrons in a soft tissue phantom. The source simulation was verified in a previous study on BNCT [26] and the same input files of the source simulations were used herein for calculation of macroscopic dose enhancement for various diameters of ${ }^{10} \mathrm{~B}$ nanoparticles (with 25,50 , and $100 \mathrm{~nm}$ diameters) with different concentrations (100, 200, and $500 \mathrm{ppm}$ ). Macroscopic dose enhancement for various neutron cross-section libraries (ENDF/B-VII.1, JEFF-3.2, JENDL-4.0u2 and CENDL-3.1) with $50 \mathrm{~nm}{ }^{10} \mathrm{~B}$ nanoparticles was also calculated and compared via the simulations performed using MCNPX Monte Carlo code. Furthermore, macroscopic dose enhancement in presence of ${ }^{10} \mathrm{~B}$ nanoparticles for tumor distances of 1,2 , and $3 \mathrm{~cm}$ from the ${ }^{252} \mathrm{Cf}$ source's center was investigated.

\section{${ }^{252} \mathrm{Cf}$ source geometry}

A ${ }^{252} \mathrm{Cf}$ (Applicator Tube [AT] model) source has been used as the source of neutrons $[22,25]$. AT ${ }^{252} \mathrm{Cf}$ source model is composed of a primary capsule, a secondary capsule, and an active core. The cylindrical active element is made of $\mathrm{Pd}: \mathrm{Cf}_{2} \mathrm{O}_{3}$ ceramic metal with $15.0 \mathrm{~mm}$ length and $1.23 \mathrm{~mm}$ diameter having mass density of $12.0 \mathrm{~g} / \mathrm{cm}^{3}$. The primary capsule is composed of a $\mathrm{Pt} / \mathrm{Ir}-10 \%$ alloy (with mass density of $21.51 \mathrm{~g} / \mathrm{cm}^{3}$ ) with inner diameter of 1.35 $\mathrm{mm}$ and outer diameter of $1.75 \mathrm{~mm}$. The inner and outer lengths of the primary capsule are 15.50 and $17.78 \mathrm{~mm}$, respectively. The secondary capsule has the same composition but its inner diameter is $1.80 \mathrm{~mm}$ and its outer diameter is $2.80 \mathrm{~mm}$, while its inner and outer lengths are $17.82 \mathrm{~mm}$ and $23.14 \mathrm{~mm}$, respectively. The inner and outer capsules have rounded with weld ends. There is a Bodkin eyelet with $0.635 \mathrm{~mm}$ diameter in the outer capsule [22]. Figure 1 illustrates the geometry of this source model.

\section{${ }^{252} \mathrm{Cf}$ source simulation verification}

In this study, the validated simulations of AT ${ }^{252} \mathrm{Cf}$ source presented in reference [26] were used. While 


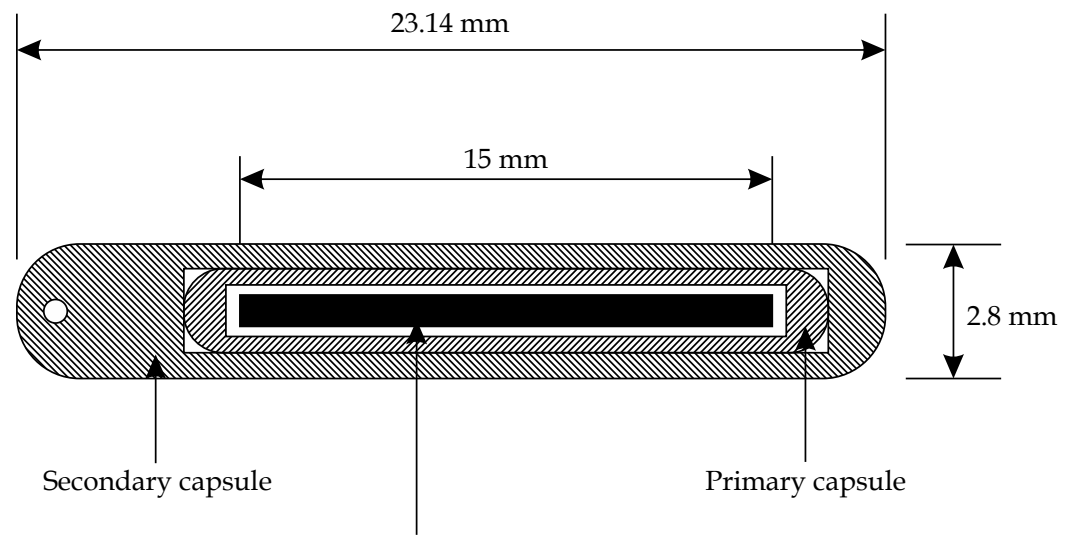

Active core

Fig. 1. Geometry of applicator tube ${ }^{252} \mathrm{Cf}$ source used in this study

the validations were not repeated in the present study, a summary of the source simulation in reference 26 is presented. MCNPX (version 2.6.0) [27] Monte Carlo code has been used for simulation of the ${ }^{252} \mathrm{Cf}$ source. Dosimetric parameters of the source, including air kerma strength, dose rate constant, and radial dose function have been calculated based on the formalism presented in the updated report by American Association of Physicists in Medicine (AAPM) Task Group No. 43 (TG-43U1) [28].

In addition to TG-43U1 parameters, total dose rate per microgram of the ${ }^{252} \mathrm{Cf}$ source $(\mathrm{cGy} /(\mathrm{h}-\mu \mathrm{g}))$ has been also calculated. Total dose equals to the sum of dose contributions from primary photons from ${ }^{252} \mathrm{Cf}$, neutrons and secondary photons induced from neutron interactions. The photon spectrum of the ${ }^{252} \mathrm{Cf}$ source has photon energies in the range of $10 \mathrm{keV}-10 \mathrm{MeV}$ [29]. One microgram of ${ }^{252} \mathrm{Cf}$ source emits $2.314 \times 10^{6}$ fast neutrons/s [30] and also has a photon yield of about $1.3 \times 10^{7}$ photons/s [31]. Maxwellian fission distribution has been utilized as the distribution for the neutrons emitted by the ${ }^{252} \mathrm{Cf}$ source. The neutron spectrum emitted by ${ }^{252} \mathrm{Cf}$ source has an average energy of $2.1 \mathrm{MeV}$ and also the most probable energy of $\sim 0.7 \mathrm{MeV}$ [32]. In calculation of dose rate constant, radial dose function, and total dose rate, a water phantom with $15 \mathrm{~cm}$ radius was defined. F6 tally was applied to score the photon and neutron doses in the verification step. For calculation of the total dose, two input files have been run: the first input file included a photon source, defined with the photon spectrum emitted by ${ }^{252} \mathrm{Cf}$ source, and the photon tally was computed for the purpose of calculation of the primary photon dose component; the second input file included the neutron spectrum of the ${ }^{252} \mathrm{Cf}$ source and the dose components from the neutrons and secondary photons were calculated. In calculation of dose rate, constant and radial dose function, a number of $10^{7}$ neutrons were scored and the maximum type A uncertainty in Monte Carlo calculations was $0.78 \%$. In calculation of total dose rate, a number of $2.0 \times 10^{8}$ particles were run in each input file. The type A uncertainty in the tally cells in these Monte Carlo calculations were less than or equal to $6.60 \%$ in these two types of files. Finally, in the study by Khosroabadi et al. [26], the comparison of air kerma strength, dose rate constant, radial dose function, and total dose rate has shown good agreement between the obtained data and those reported in other studies [32-34] for the AT ${ }^{252} \mathrm{Cf}$ source, which validated the simulations for this source model.

\section{Effect of diameter of nanoparticles and capture cross-section library}

In this step, the verified $\mathrm{AT}{ }^{252} \mathrm{Cf}$ source simulations from reference 26 were used for calculation of macroscopic dose enhancement for various diameters of ${ }^{10} \mathrm{~B}$ nanoparticles and various neutron cross-section libraries. MCNPX (version 2.6.0) Monte Carlo code has been utilized for this purpose. Soft tissue composition announced by the report No. 44 of International Commission on Radiation Units and Measurements (ICRU), with weight fractions of $\mathrm{H}: 0.102 ; \mathrm{C}: 0.143 ; \mathrm{O}: 0.708 ; \mathrm{N}: 0.034$; $\mathrm{Na}: 0.002 ; \mathrm{P}: 0.003 ; \mathrm{Cl}: 0.002 ; \mathrm{S}: 0.003 ; \mathrm{K}: 0.003$, and mass density of $1.06 \mathrm{~g} / \mathrm{cm}^{3}$ has been used as the phantom media [35]. Furthermore, abundances of natural isotopes of each element have been utilized in these dosimetric calculations. The soft tissue phantom had a spherical form with $15 \mathrm{~cm}$ radius, while the ${ }^{252} \mathrm{Cf}$ source has been defined, including its capsules and active core, at the center of the phantom. The phantom contained a $1 \mathrm{~cm} \times 1 \mathrm{~cm}$ $\times 1 \mathrm{~cm}$ tumor placed at $1 \mathrm{~cm}$ from the ${ }^{252} \mathrm{Cf}$ source's center in the transverse direction relative to the source's longitudinal axis. 100, 200, and 500 ppm concentrations of ${ }^{10} \mathrm{~B}$ has been considered as the capture agent inside the tumor in the form of nanoparticles. The effect of diameter of ${ }^{10} \mathrm{~B}$ nanoparticles on macroscopic dose enhancement was evaluated. For this purpose, 25, 50, and $100 \mathrm{~nm}$ diameters of ${ }^{10} \mathrm{~B}$ nanoparticles were studied. Each nanoparticle diameter and concentration has been examined in a separate simulation. The composition of the tumor included the capture medium $\left({ }^{10} \mathrm{~B}\right.$ nanoparticles) and soft tissue, and was defined as distribution of the nanoparticles in the Monte Carlo programs. ${ }^{10} \mathrm{~B}$ nanoparticles were defined as nanospheres with 25, 50, and $100 \mathrm{~nm}$ diameters using lattice cards in the MCNPX code. In this code, a lattice can be utilized as rectangular parallelepiped (RPP) surfaces for defintion of repeated structures inside a volume. ${ }^{10} \mathrm{~B}$ nanoparticles were distributed uniformly inside 
the tumor volume. Our rational for selection of 25, 50, and $100 \mathrm{~nm}$ diameters of nanoparticles was that nanoparticles ranging in diameter from $1 \mathrm{~nm}$ to $100 \mathrm{~nm}$ are usually applied in the field of nanomedicine [36]. By considering the diameter and concentration of ${ }^{10} \mathrm{~B}$ nanoparticles, the tumor was divided into cubic cells with dimensions of order of nanometer and in each cell a ${ }^{10} \mathrm{~B}$ nanoparticle was defined. The dimensions of the tally voxels were $2 \mathrm{~mm} \times 2 \mathrm{~mm} \times 2 \mathrm{~mm}$. Since the tumor was considered as a $1 \mathrm{~cm} \times 1 \mathrm{~cm} \times 1 \mathrm{~cm}$ cube, there were 5 voxels on the transverse line in the tumor. In this study, the total dose was calculated as the sum of the dose components from primary and secondary photons, neutrons, and the capture products. For calculation of dose component from neutron capture products in each voxel, the neutron flux in each voxel inside the tumor was calculated using F4 tally and then was converted to dose by multiplication of the neutron flux and kerma factor. The kerma factors of ${ }^{10} \mathrm{~B}(\mathrm{n}, \mathrm{a})$ interactions have been obtained from the following formula using the neutron capture cross-sections $(\sigma)$ :

$$
F_{n(} E_{i)}=\sum_{j, k} \sigma_{k(j)} \frac{x i}{M j} N_{A} \varepsilon_{k, j}
$$

where $F_{n}$ is kerma factor; $x_{i}$ is the mass fraction of the capture agent; $M$ is atomic mass; $N_{A}$ is Avogadro's number; and $\varepsilon$ is the delivered energy in the capture process.

For each nanoparticle diameter, two input files have been run: the first input file with a photon source (emitted by ${ }^{252} \mathrm{Cf}$ ) and photon tally scoring for the purpose of calculation of the primary photon dose component; the second input file with a neutron source (emitted by ${ }^{252} \mathrm{Cf}$ ) and the dose components from secondary photons, neutrons, and capture products were scored. The first and second input files were run for a number of $10^{8}$ particles in calculation of macroscopic dose enhancement and the maximum Monte Carlo type A uncertainty over all the situations and tally cells was $4.67 \%$. Macroscopic dose enhancement in the center of each voxel in tumor on transverse line was calculated and then macroscopic dose enhancement values were averaged. Macroscopic dose enhancement factor (MDEF) in each voxel has been determined as the total dose in the voxel with presence of ${ }^{10} \mathrm{~B}$ nanoparticles in tumor to the total dose in the same voxel without presence of ${ }^{10} \mathrm{~B}$ nanoparticles. Since the absorbed dose in radiotherapy is a macroscopic variable, it was preferred in the present study to use a macroscopic dose enhancement defintion, as it is in a number of other studies in this field. However, the nanoparticles were defined in a nanometer scale in the form of nanosphers. ENDF/B-VII.1 cross-section library

Table 1. The average macroscopic dose enhancement factor values for 25,50 and $100 \mathrm{~nm}{ }^{10} \mathrm{~B}$ nanoparticles with concentrations of 100,200 , and 500 ppm

\begin{tabular}{lccc} 
& $25 \mathrm{~nm}$ & $50 \mathrm{~nm}$ & $100 \mathrm{~nm}$ \\
\hline $100 \mathrm{ppm}$ & 1.02 & 1.03 & 1.04 \\
\hline $200 \mathrm{ppm}$ & 1.04 & 1.05 & 1.03 \\
\hline $500 \mathrm{ppm}$ & 1.10 & 1.08 & 1.10
\end{tabular}

was used in the evaluation of the effect of nanoparticle's diameter with different concentrations.

The effect of neutron capture cross-section library for neutron-boron capture interaction on MDEF for various library sources including: ENDF/B-VII.1, JEFF-3.2, JENDL-4.0u2, and CENDL-3.1 was evaluated. The capture cross-sections have been elicited from the cross-section data reported by the International Atomic Energy Agency (IAEA) webpage for $(n, \alpha)$ interaction with ${ }^{10} \mathrm{~B}$ isotope [37]. Macroscopic dose enhancement factor value and total dose rate $(\mathrm{cGy} /(\mathrm{h} \cdot \mu \mathrm{g}))$ were calculated for the cross-section libraries for $500 \mathrm{ppm}$ concentration of $50 \mathrm{~nm}$ diameter ${ }^{10} \mathrm{~B}$ nanoparticles. The details of these simulations (eg. the number of input files, the number of particle histories, etc.) were similar to those mentioned above in the evaluation of the effect of diameter of nanoparticles. The maximum Monte Carlo type A uncertainty over all the situations and tally cells was $4.56 \%$.

Beside to the distance of $1 \mathrm{~cm}$ for tumor from the source center, other distances $(2 \mathrm{~cm}$ and $3 \mathrm{~cm})$ were examined as well. For this purpose, the tumour was defined at distances of $2 \mathrm{~cm}$ and $3 \mathrm{~cm}$ from the center of the ${ }^{252} \mathrm{Cf}$ source at the transverse direction, while each distance was defined in a separate simulation. In these two cases, the diameter of the nanoparticles was $50 \mathrm{~nm}$ and the concentration was $500 \mathrm{ppm}$. Other simulation details were the same as that described above for $1 \mathrm{~cm}$ tumor distance.

Macroscopic dose enhancement in presence of 500 ppm ${ }^{10} \mathrm{~B}$ nanoparticles with $50 \mathrm{~nm}$ diameters for tumor distances of 1,2 , and $3 \mathrm{~cm}$ from the ${ }^{252} \mathrm{Cf}$ source's center was investigated as well. For distances of $2 \mathrm{~cm}$ and $3 \mathrm{~cm}$, $4 \times 10^{8}$ particles were scored in each input program and the maximum type A uncertainty in the calculations for these programs was $4.96 \%$.

\section{Results}

The average MDEF values for 25, 50 and $100 \mathrm{~nm}{ }^{10} \mathrm{~B}$ nanoparticles with concentrations of 100, 200 and 500 ppm are listed in Table 1. The average values have been obtained as the averages of MDEFs over the five voxels on the transverse line inside the tumor.

The plots of MDEF and the total dose rate values (cGy/ $(\mathrm{h} \cdot \mu \mathrm{g})$ ) inside the tumor versus radial distance from the ${ }^{252} \mathrm{Cf}$ source are shown in Figure 2 for different diameters of ${ }^{10} \mathrm{~B}$ nanoparticles. In this figure, parts (A) and (B) are related to MDEF and total dose rate, respectively.

The average MDEF values for ENDF/B-VII.1, JEFF-3.2, JENDL-4.0u2, CENDL-3.1 capture cross-section libraries for 500 ppm concentrations of ${ }^{10} \mathrm{~B}$ with $50 \mathrm{~nm}$ diameter was obtained equal to 1.08. This value was obtained the same for the four capture cross-section libraries evaluated. The plots of the MDEF and the total dose rate values (cGy/(h- $\mu \mathrm{g})$ ) inside the tumor were illustrated in Figure 3 for the four capture cross-section libraries. In these plots, parts (A) and (B) correspond to MDEF and total dose rate, respectively.

For tumor distances of 1,2 , and $3 \mathrm{~cm}$ from the ${ }^{252} \mathrm{Cf}$ source's center, the average MDEF values for $50 \mathrm{~nm}{ }^{10} \mathrm{~B}$ nanoparticles with 500 ppm concentration were obtained equal to $1.08,1.18$, and 1.27 , respectively. 
A

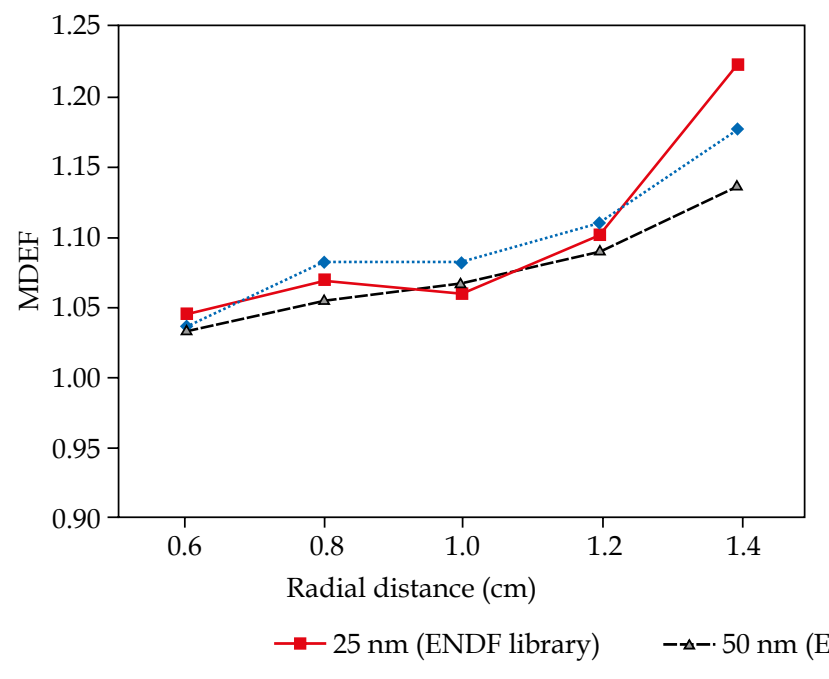

B

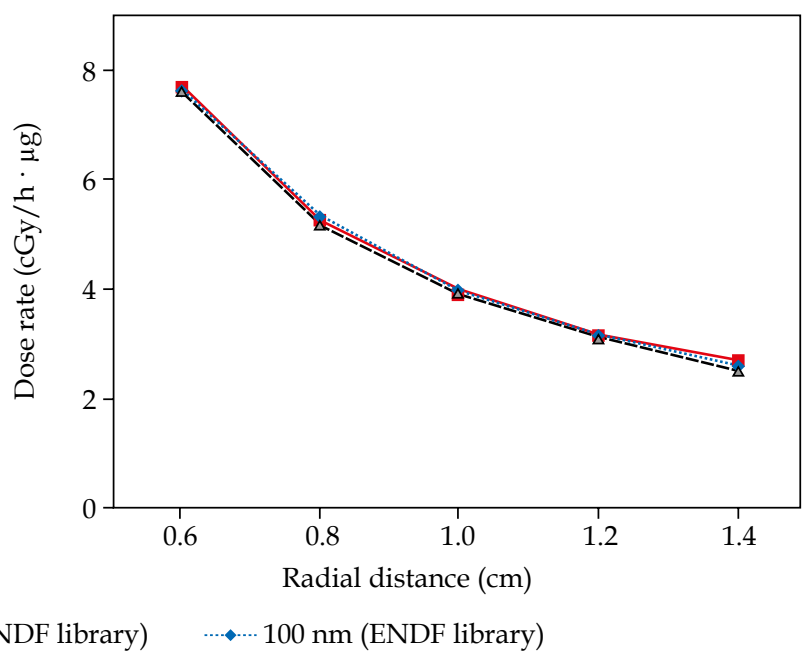

Fig. 2. Macroscopic dose enhancement factor (part A) and total dose rate (part B) in tumor for 25,50 , and 100 nm ${ }^{10} \mathrm{~B}$ nanoparticles with $500 \mathrm{ppm}$ concentration of ${ }^{10} \mathrm{~B}$ nanoparticles (with ENDF capture cross-section library)

A

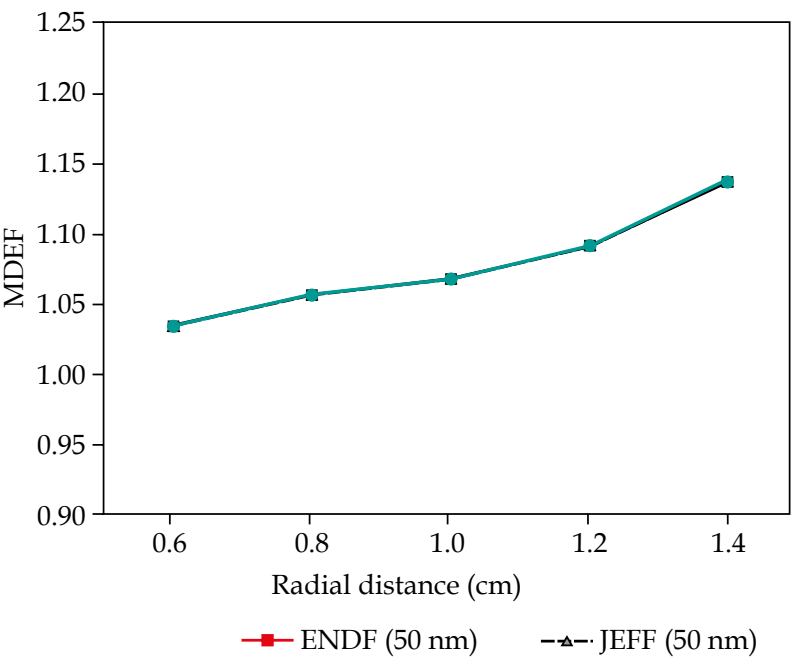

B

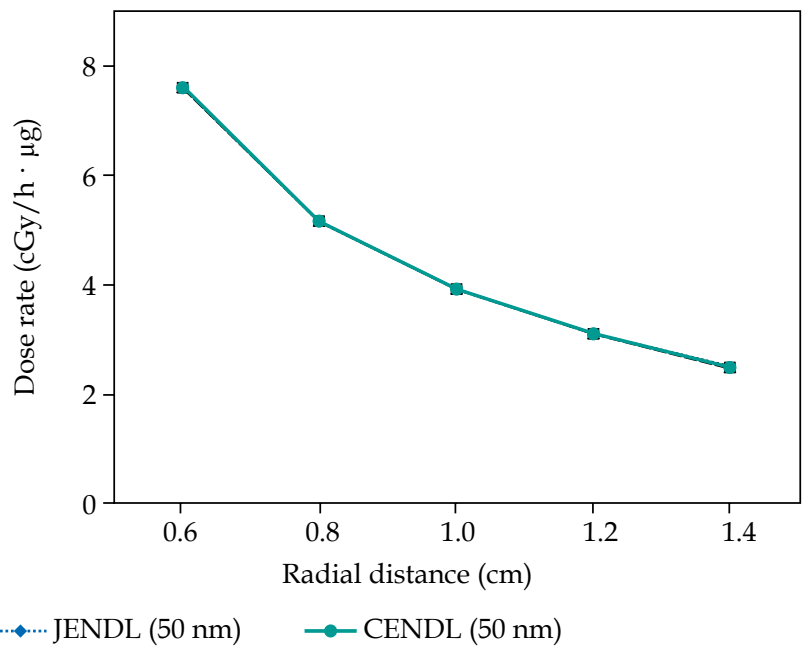

Fig. 3. Macroscopic dose enhancement factor (part A) and total dose rate (part B) in tumor for ENDF, JEFF, JENDL, and CENDL capture cross-section libraries with 500 ppm concentration of ${ }^{10} \mathrm{~B}$ nanoparticles (with $50 \mathrm{~nm}$ diameter)

The percentage contributions of each dose component (primary photons, secondary photons, neutrons, and boron capture products) from the total dose in the central voxel inside the tumor without and with 500 ppm concentration of ${ }^{10} \mathrm{~B}$ nanoparticles are listed in Table 2.

\section{Discussion and conclusions}

In this study, the effect of diameter of ${ }^{10} \mathrm{~B}$ nanoparticles with different concentrations and the effect of various neutron capture cross-section libraries on macro-

Table 2. The percentage contributions (\%) of dose components of the total dose without and with 500 ppm boron nanoparticles in tumor. The values are related to the central voxel in the tumor

\begin{tabular}{lcccc} 
& Primary photon & Secondary photon & Neutron & Boron capture products \\
\hline Without nanoparticles & 29.45 & 0.80 & 69.75 & - \\
\hline $25 \mathrm{~nm}$ nanoparticles & 27.75 & 0.75 & 64.05 & 7.44 \\
\hline $50 \mathrm{~nm}$ nanoparticles & 27.59 & 0.75 & 64.56 & 7.10 \\
\hline $100 \mathrm{~nm}$ nanoparticles & 27.21 & 0.76 & 65.24 & 6.79
\end{tabular}


scopic dose enhancement in BNCT were investigated. The obtained results (Table 1 ) indicates that various ${ }^{10} \mathrm{~B}$ nanoparticle diameters have no dominant effect on average MDEF value in tumor in various concentrations. On the other hand, the data in this table imply that the average MDEF values grows with increase of concentration for ${ }^{10} \mathrm{~B}$ nanoparticles with same diameter. This effect confirms the results of a previous study in this field on BNCT with various ${ }^{10} \mathrm{~B}$ nanoparticles [26]. It should be noticed that from only physical point of view, various nanoparticle diameters have no dominant effect on average MDEF, however, for detailed evaluation of the effects of nanoparticle diameters, biological effects should also be considered. For the purpose of incorporation of biological effect, it is normal that each dose component (from photons, neutrons, and capture products) is multiplied with the corresponding relative biological effectiveness (RBE) of that particle. Various RBE values have been reported for photons and neutrons in literatures. As an example, values of 1.0 and 3.0 were reported by Kawabata et al. for photons and neutrons, respectively [38]. Various compounds were proposed for delivery of boron to tumor: borono-phenylalanine $\left({ }^{10} \mathrm{BPA}\right)$ and sodium mercaptoundecahydro-dodecaborate $\left(\mathrm{Na}_{2}{ }^{10} \mathrm{~B}_{12} \mathrm{H}_{11} \mathrm{SH}\right.$ :BSH). The distribution of these compounds is not homogeneous in tissue. Therefore, another factor is applied in dose calculations in the field of BNCT, which is compound biological effectiveness (CBE). This factor incorporates the effect of both RBE and boron distribution in the dose calculations. Kawabata et al. [38] reported a CBE of 3.8 for the ${ }^{10} B(n, a)$ ${ }^{7} \mathrm{Li}$ reaction in BPA. This value is specific to BPA and cannot be used for other compounds. Since the RBE and CBE for the neutron-alpha interactions in the ${ }^{10} \mathrm{~B}$ nanoparticles used in this study are not available, the MDEFs have been only calculated for the physical dose values. Macroscopic dose enhancement factor obtained from the product of the $\mathrm{CBE}$ and the physical dose will differ with the MDEF calculated from the physical dose alone. It is obvious that the RBE weighted MDEF values will depend on the RBE values, which are used for various nanoparticle diameters in the calculations. Since various nanoparticle diameters have different cellular penetration, therefore they have different distributions and RBEs. In our study, one notes that the RBE values of ${ }^{10} \mathrm{~B}$ nanoparticles with various diameters are not available, therefore only physical dose enhancements were calculated for different diameters of nanoparticles. It is predicted that taking into account the biological effects for various nanoparticle diameters will result in different macroscopic dose enhancements when compared to those obtained herein.

Considering the effect of diameter of nanoparticles and biological aspects on macroscopic dose enhancement in other radiotherapy modalities may be useful. According to the definition by American Society for Testing and Materials standard, nanoparticles are particles ranging in diameter from 1 to $100 \mathrm{~nm}$ in two or three dimensions [36]. It has been shown that macroscopic dose enhancement inside a tumor is influenced by the beam's quality, concentration, diameter of nanoparticles, etc. [18].

The overall particle's size must be large enough to avoid renal clearance but small enough to evade uptake by reticuloendothelial system [39]. It has also been shown that for nanoparticles smaller than $40 \mathrm{~nm}$ in diameter, both the half-life and biodistribution of nanoparticles are influenced by the coating material rather than the mean size [40].

In experimental studies on GNPs, the size of GNPs has been shown to be as a factor effecting on radiosensitization [41]. Literatures have reported that smaller GNPs are more effective [42], while the others suggest an opposite effect [43]. In an in vitro study, Chithrani et al. investigated the impact of GNP diameter, radiation energy, and concentration on radiosensitisation in Hela cells. It has been evident that GNPs with $50 \mathrm{~nm}$ diameter have higher radiosensitization enhancement factor (REF) than $14 \mathrm{~nm}$ or $74 \mathrm{~nm}$ GNPs, although the highest amount of gold was delivered with $74 \mathrm{~nm}$ nanoparticles [44]. The reason for this effect was due to higher gold loading in nanoparticles with larger particle sizes [45]. A smaller diameter GNPs $(15 \mathrm{~nm})$ showed higher distribution in tissues compared to larger particle diameters. Hillyer and Albrecht [46] evaluted the gastrointestinal uptake and tissue/organ distribution of $4,10,28$, and $58 \mathrm{~nm}$ diameter GNPs. The smallest size GNPs (with $4 \mathrm{~nm}$ diameter) showed greater uptake in various tissues. Gold was not detected in most of the tissues evaluated following oral administration of $58 \mathrm{~nm}$ diameter colloidal gold. In a study by Jiang et al. [47] it was confirmed that the optimal diameter for nanoparticle cellular entry was $40-50 \mathrm{~nm}$.

Additionally to the above studies, there are also Monte Carlo studies carried out in the field of dose enhancement by GNPs. Ghorbani et al. [17] evaluated the effect of diameter of gold nanoparticles with 50, 100, and $200 \mathrm{~nm}$ diameters on macroscopic dose enhancement in brachytherapy with photon sources. Their results indicated that with gold nanoparticles of larger diameter, the amount of macroscopic dose enhancement is higher inside the tumor. In another study, Pakravan et al. [18] investigated the effect of size of nanoparticles with 25-200 nm diameters on macroscopic dose enhancement in tumor with a $6 \mathrm{MV}$ photon beam. It has been found that there is a negligible relation between the macroscopic dose enhancement and nanoparticle's diameter in this photon energy. It was evident that for high energy photons, the macroscopic dose enhancement is lower affected by the diameter of nanoparticles than their concentration. In a study by Leung et al. [48], the interaction of X-rays with GNPs with 2, 50, and $100 \mathrm{~nm}$ diameters has been simulated, and the results showed that nanoparticles with larger diameters provide greater dose values. Lechtman [49] carried out a Monte Carlo study on the role of nanoparticle's diameter, photon energy, intracellular location, and concentration of nanoparticles on the radiosensitization to determine the optimal clinical use of GNPs. He found that GNPs' size can strongly impress radiosensitization due to the size dependent cellular uptake. Similarly Chithrani et al. [44] found that GNPs of $50 \mathrm{~nm}$ were a desired size for accumulation and retention within Hela cells.

In a clinical study, Mortensen et al. [50] used boron carbide nanoparticles as a novel agent in T cell-guided BNCT. They found that desired size distribution for boron carbide nanoparticles is $73 \mathrm{~nm}$. The above mentioned 
effects are related a combination of photon sources and gold nanoparticels. It is obvious that they may not be extended simply for a neutron source and ${ }^{10} \mathrm{~B}$ nanoparticles in BNCT applications. However, similar phenomena may be expected in some cases in application of ${ }^{10} \mathrm{~B}$ nanoparticles in BNCT. In other words, additionally to the physical macroscopic dose enhancement seen for ${ }^{10} \mathrm{~B}$ nanoparticles, other biological aspects should be sought and evalualted as well for ${ }^{10} \mathrm{~B}$ nanoparticles with various diameters.

While the MDEF values have different decreasing or increasing trends in the tumor for various diameters of nanoparticles and capture cross-section libraries (Fig. 2 and 3$)$, the dose rate values (cGy/(h/ $\mu \mathrm{g}))$ are decreasing in tumor with the radial distance from the ${ }^{252} \mathrm{Cf}$ source. This decreasing trend in dose rate is due to inverse square law with distance and the attenuation in phantom material. It can be concluded from this effect that in treatment planning in neutron capture therapy, various points inside a tumor will experience different macroscopic dose enhancements and this effect should be considered. However, it is clear that MDEF differs from dose rate value and the MDEF only cannot be the sole criterion in clinical considerations.

The average MDEF values were the same for various capture cross-section libraries (for 500 ppm concentration of ${ }^{10} \mathrm{~B}$ with $50 \mathrm{~nm}$ diameter the MDEF was obtained equal to 1.08). Furthermore, the trends of the MDEF values inside tumor are the same for different capture cross-section libraries (Fig. 3). In summary, while there may be local differences in various neutron capture cross-section libraries, it has been shown that using different cross-section libraries in BNCT calculations do not show a difference in MDEF and total dose rate values.

The average MDEF values for tumor distances of 1,2 , and $3 \mathrm{~cm}$ from the ${ }^{252} \mathrm{Cf}$ source's center are $1.08,1.18$, and 1.27 , respectively. As it can be evident from these data, there is an increasing trend for average MDEF with the tumor distance. This effect may be due to the change in the energy spectrum of the neutrons (or photons) with the distance in the phantom. It is obvious that when the neutrons (and photons) emitted by the ${ }^{252} \mathrm{Cf}$ source traverse in the phantom, the interactions between the particles and the phantom material will change the energy spectrum. Therefore, the number of thermal neutrons will be different at various distances from the source. This will effect on the number of capture interactions with ${ }^{10} \mathrm{~B}$ nanoparticles at various distances. A tumor at furthere distance from the source experience a higher average MDEF and this will be an advantage for this type of tumor in BNCT. On the other hand, the dose is different from MDEF and in this case the normal tissue between the source and tumor receives a larger dose due to the steep dose gradient in the vicinity of the source.

Based on the data in Table 2 (for concentration of 500 ppm), the maximum and minimum doses that will effect the total dose rate in tumor are neutron and secondary photon doses, respectively. Furthermore, according to these data it can be concluded, while there was not a linear relationship between the nanoparticles' diameter and the MDEF value, for a larger diameter of nanoparticles the boron-related dose component reduces in some extent. It can be also mentioned that in BNCT calculations, the total dose should be involved and considering only the neutron dose will introduce underestimations in dose calculations.

According to the results of this study, it can be concluded that the selection of optimum diameter of ${ }^{10} \mathrm{~B}$ nanoparticles needs to additional studies on biological aspects. In other words, it is necessary to take into account the biological effects differences in dose calculations. For this purpose, each dose component should be multiplied with the corresponding RBE of each particle. It can be also concluded that using various capture cross-section libraries result to the same average MDEF values.

There are other limitations in the present study. Lack of $\mathrm{RBE}$ for various diameters of ${ }^{10} \mathrm{~B}$ nanoparticles is one of the limitations. A radiobiologic study on this subject can be a basic support for dose enhancement studies on various ${ }^{10} \mathrm{~B}$ nanoparticle diameters. Furthermore, a uniform distribution of ${ }^{10} \mathrm{~B}$ was defined in the tumor, while in a real situation the distribution will be nonuniform. The obtained macroscopic dose enhancement results are geometry-specific and it is obvious that by having a different source and tumor geometry, the results will be different in some extent. Since absorbed dose is a macroscopic variable, it was preferred to use $2 \mathrm{~mm} \times 2 \mathrm{~mm} \times 2 \mathrm{~mm}$ voxel sizes in calculations of dose enhancements. This method was used in a number of previous studies in the field of dose enhancment in radiotherapy $[17,18,26]$. On the other hand, Tsiamas et al. [51] and Zygmanski et al. [52] showed that the nanoscopic dose enhancement factor is practically unity after 1-2 microns. Using a voxel of $2 \mathrm{~mm} \times 2 \mathrm{~mm}$ $\times 2 \mathrm{~mm}$ means that the calculated dose enhancement factor is lower than that it is in the reality as the total dose to soft tissue would not be smaller than the dose when nanoparticles are present. Therefore, performing a similar study, as it is in the present one, on dose enhancment by various diameters of ${ }^{10} \mathrm{~B}$ nanoparticles at a micro scale will be as a complementary to the present results. This is especially true for a BNCT study as the boron capture products have short ranges in soft tissue. Another aspect of the present study, which should be considered in more details, is the definition of concentration in evaluation of the effect of nanoparticle's diameter. Many studies have used concentration but the calculated dose enhancement factor for these studies is more a macroscopic dose rather than a nanoscopic one. In the nanoscopic scale, quantities such as concentration, dose etc. have different meanings. Furtheremore, the $\mathrm{AT}^{252} \mathrm{Cf}$ source is a low dose rate neutron source and primary sources with higher neutron fluxes can provide higher number of neutrons and therefore higher dose enhancment values. These items can be as subjects of further research in the field of BNCT.

\section{Acknowledgments}

The authors would like to thank Mashhad University of Medical Sciences (Mashhad, Iran) for financial support of this work.

\section{Disclosure}

Authors report no conflict of interest. 


\section{References}

1. Taylor HJ. The disintegration of boron by neutrons. Proc Phys Soc 1935; 47: 873-876.

2. Barth RF, Vicente M, Harling OK et al. Current status of boron neutron capture therapy of high grade gliomas and recurrent head and neck cancer. Radiat Oncol 2012; 7: 1-21.

3. Cerullo N, Esposito J, Daquino G. Spectrum shaping assessment of accelerator-based fusion neutron sources to be used in BNCT treatment. Nucl Instrum Methods Phys Res B 2004; 213: 641-645.

4. Barth RF, Coderre JA, Vicente MGH et al. Boron neutron capture therapy of cancer: current status and future prospects. Clin Cancer Res 2005; 11: 3987-4002.

5. Diaz AZ, Coderre JA, Chanana AD et al. Boron neutron capture therapy for malignant gliomas. Ann Med 2000; 32: 81-85.

6. Fukuda $\mathrm{H}$, Honda $\mathrm{C}$, Wadabayashi $\mathrm{N}$ et al. Pharmacokinetics of ${ }^{10} \mathrm{~B}$-p-boronophenylalanine in tumours, skin and blood of melanoma patients: a study of boron neutron capture therapy for malignant melanoma. Melanoma Res 1999; 9: 75-84.

7. Nano R, Barni S, Chiari P et al. Efficacy of boron neutron capture therapy on liver metastases of colon adenocarcinoma: optical and ultrastructural study in the rat. Oncol Reports 2004; 11: 149-154.

8. Dagrosa MA, Crivello M, Perona M et al. First evaluation of the biologic effectiveness factors of boron neutron capture therapy (BNCT) in a human colon carcinoma cell line. Int J Radiat Oncol Biol Phys 2011; 79: 262-268.

9. Kato I, Ono K, Sakurai Y et al. Effectiveness of BNCT for recurrent head and neck malignancies. Appl Radiat Isot 2004; 61: 1069-1073.

10. Nakamura H, Kirihata M. Boron Compounds: New Candidates for Boron Carriers in BNCT. In: Sauerwein WA, Wittig A, Moss R, Nakagawa Y. Neutron Capture Therapy: Principles and Applications. Springer, Berlin Heidelberg 2012; pp. 99-116.

11. Kato I, Ono K, Sakurai Y et al. Effectiveness of BNCT for recurrent head and neck malignancies. Appl Radiat Isot 2004; 61: 1069-1073.

12. Cuenca AG, Jiang H, Hochwald SN et al. Emerging implications of nanotechnology on cancer diagnostics and therapeutics. Cancer 2006; 107: 459-466.

13. Rao J. Shedding light on tumours using nanoparticles. ACS Nano 2008; 2: 1984-1986.

14. Langereis S, Keupp J, van Velthoven JL et al. A temperature-sensitive liposomal ${ }^{1} \mathrm{H}$ CEST and ${ }^{19} \mathrm{~F}$ contrast agent for MR image-guided drug delivery. J Am Chem Soc 2009; 131: 1380-1381.

15. Perrault SD, Walkey C, Jennings $\mathrm{T}$ et al. Mediating tumour targeting efficiency of nanoparticles through design. Nano Lett 2009; 9: 1909-1915.

16. Lee JE, Lee N, Kim H et al. Uniform mesoporous dye-doped silica nanoparticles decorated with multiple magnetite nanocrystals for simultaneous enhanced magnetic resonance imaging, fluorescence imaging, and drug delivery. J Am Chem Soc 2009; 132: 552-557.

17. Ghorbani M, Pakravan D, Bakhshabadi M et al. Dose enhancement in brachytherapy in the presence of gold nanoparticles: a Monte Carlo study on the size of gold nanoparticles and method of modelling. Nukleonika 2012; 57: 401-406.

18. Pakravan D, Ghorbani M, Momennezhad M. Tumour dose enhancement by gold nanoparticles in a $6 \mathrm{MV}$ photon beam: a Monte Carlo study on the size effect of nanoparticles. Nukleonika 2013; 58: 275-280.

19. Ghassoun J, Mostacci D, Molinari V et al. Detailed dose distribution prediction of Cf-252 brachytherapy source with boron loading dose enhancement. Appl Radiat Isot 2010; 68 : 265-270.
20. Rivard MJ, Zamenhof RG. Moderated ${ }^{252}$ Cf neutron energy spectra in brain tissue and calculated boron neutron capture dose. Appl Radiat Isot 2004; 61: 753-757.

21. Sumitani S, Nagasaki Y. Boron neutron capture therapy assisted by boron-conjugated nanoparticles. Polym J 2012; 44: 522-530.

22. Hwang KC, Lai PD, Chiang C-S et al. Neutron capture nuclei-containing carbon nanoparticles for destruction of cancer cells. Biomaterials 2010; 31: 8419-8425.

23. Porras I. Sulfur-33 nanoparticles: A Monte Carlo study of their potential as neutron capturers for enhancing boron neutron capture therapy of cancer. Appl Radiat Isot 2011; 69: 1838-1841.

24. Badikov SA, Zhenpeng $\mathrm{Ch}$, Carlson $\mathrm{AD}$ et al. International evaluation of neutron cross-section standards. IAEA Report 2007, STI/PUB/1291, Vienna, Austria.

25. Rivard MJ, Wierzbicki JG, Van den Heuvel F, et al. Clinical brachytherapy with neutron emitting 252Cf sources and adherence to AAPM TG-43 dosimetry protocol. Med Phys 1999; 26: 87-96.

26. Khosroabadi M, Ghorbani M, Rahmani F et al. Neutron capture therapy: a comparison between dose enhancement of various agents, nanoparticles and chemotherapy drugs. Australas Phys Eng Sci Med 2014; 37: 541-549.

27. Pelowitz DB. MCNPX user's manual, LA-CP-07-1473 Version 2.6.0. Los Alamos National Laboratory, 2008.

28. Rivard MJ, Coursey BM, DeWerd LA et al. Update of AAPM task group no. 43 report: A revised AAPM protocol for brachytherapy dose calculations. Med Phys 2004; 31: 633-674.

29. Kelm RS. In-water neutron and gamma dose determination for a new Cf-252 brachytherapy source. Available at: http:/ / smartech.gatech.edu/bitstream/1853/28121/1/kelm _ robert_s_200905_mast.pdf.; accessed Sept 2, 2013, Georgia Institute of Technology, 2009.

30. Martin RC, Miller JH. Applications of Californium-252 Neutron Sources in Medicine, Research, and Industry. Americas Nuclear Energy Symposium (ANES 2002), Miami, Florida, October 16-18.

31. Fantidis J, Potolias C, Vordos N et al. Optimization study of a transportable neutron radiography system based on a ${ }^{252} \mathrm{Cf}$ neutron source. Moldavian J Phys Sci 2011; 10: 121-131.

32. Melhus CS, Rivard MJ. Clinical brachytherapy dosimetry parameters and mixed-field dosimetry for a high dose rate Cf252 brachytherapy source. American Nuclear Society Topical Meeting in Monte Carlo, Chattanooga, 2005.

33. Krishnaswamy V. Calculated depth dose tables for californium-252 sources in tissue. Phys Med Biol 1972; 17: 56-63.

34. Colvett R, Rossi H, Krishnaswamy V. Dose distributions around a californium-252 needle. Phys Med Biol 1972; 17: 356364.

35. ICRU. ICRU Report No. 44, Tissue substitutes in radiation dosimetry and measurement. ICRU, Bethesda 1989.

36. ASTM International. E 2456-06 Terminology for nanotechnology; http://ethics.iit.edu/NanoEthicsBank/node/22, Accessed May, 2014, ASTM International, 2006.

37. IAEA. http://www-nds.iaea.org/exfor/endf.htm; accessed Sept 2, 2013.

38. Kawabata S, Miyatake S, Kuroiwa T et al. Boron neutron capture therapy for newly diagnosed glioblastoma. J Radiat Res 2009; 50: 51-60.

39. Sun C, Lee JS, Zhang M. Magnetic nanoparticles in MR imaging and drug delivery. Adv Drug Deliv Rev 2008; 60: $1252-$ 1265.

40. Briley-Saebo K, Bjornerud A, Grant D et al. Hepatic cellular distribution and degradation of iron oxide nanoparticles following single intravenous injection in rats: implications for magnetic resonance imaging. Cell Tissue Res 2004; 316: 315-323. 
41. Chithrani DB, Jelveh S, Jalali F et al. Gold nanoparticles as radiation sensitizers in cancer therapy. Radiat Res 2010; 173: 719-728.

42. Butterworth K, Wyer J, Brennan-Fournet M et al. Variation of strand break yield for plasmid DNA irradiated with high-Z metal nanoparticles. Radiat Res 2008; 170: 381-387.

43. Brun E, Sanche L, Sicard-Roselli C. Parameters governing gold nanoparticle X-ray radiosensitization of DNA in solution. Colloids Surf B Biointerfaces 2009; 72: 128-134.

44. Chithrani B, Ghazani A, Chan W. Determining the size and shape dependence of gold nanoparticle uptake into mammalian cells. Nano Lett 2006; 6: 662-668.

45. Sonavane G, Tomoda K, Makino K. Biodistribution of colloidal gold nanoparticles after intravenous administration: effect of particle size. Colloids Surf B Biointerfaces 2008; 66: 274-280.

46. Hillyer JF, Albrecht RM. Gastrointestinal persorption and tissue distribution of differently sized colloidal gold nanoparticles. J Pharm Sci 2001; 90: 1927-1936.

47. Jiang W, Kim BY, Rutka JT et al. Nanoparticle-mediated cellular response is size-dependent. Nat Nano 2008; 3: 145-150.

48. Leung MK, Chow JC, Chithrani BD et al. Irradiation of gold nanoparticles by x-rays: Monte Carlo simulation of dose enhancements and the spatial properties of the secondary electrons production. Med Phys 2011; 38: 624-631.

49. Lechtman ES. A Monte Carlo-based model of gold nanoparticle radiosensitization. Ph. D. Thesis in Medical Biophysics, https://tspace.library.utoronto.ca/bitstream/1807/43632/ 3/Lechtman_Eli_S_201311_PhD_thesis.pdf; accessed May, 2014, University of Toronto; 2013.

50. Mortensen MW, Sorensen PG, Bjorkdahl O et al. Preparation and characterization of Boron carbide nanoparticles for use as a novel agent in $\mathrm{T}$ cell-guided boron neutron capture therapy. Appl Radiat Isot 2006; 64: 315-324.

51. Tsiamas P, Liu B, Cifter F et al. Impact of beam quality on megavoltage radiotherapy treatment techniques utilizing gold nanoparticles for dose enhancement. Phys Med Biol 2013; 58: 451-464.

52. Zygmanski P, Liu B, Tsiamas P et al. Dependence of Monte Carlo microdosimetric computations on the simulation geometry of gold nanoparticles. Phys Med Biol 2013; 58: 79617977. 